\title{
Research on Wireless Sensor Network Routing Protocol Based on Energy Equilibrium
}

\author{
ZHANG Linsheng, a \\ ${ }^{1}$ School of Communication Engineering, Chongqing College of electronic engineering, Chongqing, \\ 401331 \\ a56016681@qq.com
}

Keywords: Wireless Sensor Networks; Energy Equalization, Low Power

\begin{abstract}
Wireless sensor network is composed of a large number of distributed in a specific area, with the calculation and communication capabilities of small sensor nodes densely distributed in the unattended monitoring area and can be completed according to the environment independent task of intelligent autonomous monitoring and control network system. Wireless sensor network node energy is limited, the need for efficient energy-efficient routing protocol to reduce network energy consumption. The energy equalization clustering protocol constructs clusters of varying sizes according to the distance from the node from the convergence point, the residual energy and the distribution density. The energy campaign is based on the node's energy level relative to the surrounding candidate, avoiding the need for low energy nodes to be elected as energy. The multi hop routing algorithm is designed to consider the optimal energy consumption of the link and the energy balance between the nodes. The routing protocol significantly balances the energy consumption of nodes in the network and extends the network lifetime.
\end{abstract}

\section{Theoretical Introduction of Wireless Sensor Network}

Wireless sensor network is a new type of wireless network, which consists of a series of cheap, low power and sensor, data processing and short-range wireless communication function of the sensor node composed of multiple sensor nodes in a self-organized way to form a certain And collects and processes the information of the monitoring object in the network coverage area and transmits the information to the monitor.The wireless sensor network can realize real-time data monitoring of the target area by randomly deploying it in the monitoring area, so as to realize the real-time data monitoring of the target area.

The planar and hierarchical types are the two kinds of network topologies which are mainly used in wireless sensor networks. The nodes in the planar wireless sensor networks are completely equal and they are perceived by the MAC protocol under the control of multi-hop forwarding between nodes data is transmitted to the monitoring base station. The hierarchical wireless sensor network adopts the clustering algorithm to divide the nodes in the network into several clusters, and processes the data of the nodes in the cluster. Compared with the planar network topology, Nodes with high number of nodes and high density, the hierarchical network topology can reduce the transmit power of the nodes in the cluster, reduce the number of nodes in the competing channels, reduce the energy consumption of the nodes, and provide more routing information for the nodes, Thus effectively improve the MAC protocol and routing protocol efficiency ${ }^{[1]}$.

The hierarchical network topology also has good scalability and can provide a good basis for data fusion, time synchronization and target location in wireless sensor networks. The important design goal of wireless sensor networks is to balance the load and energy of network nodes However, from the previous analysis we can see that the existing wireless sensor network clustering algorithm prevalent cluster head node load and energy consumption is not balanced, and this imbalance will lead to some nodes energy consumption is too fast, so as to shorten the network survival time. 


\section{Wireless Network Model}

Hundreds of cheap, low-power sensors are self-organized into wireless sensor networks. They cooperate in detecting and sensing a wide range of environmental information in the area covered by the network. They can be widely used in industrial control, intelligent home, urban transportation and other fields. Simple data collection application scenarios such as environmental testing, industrial testing, etc., more use of single Sink network organization form, the entire system reliability, scalability by the Sink node constraints, the network can't handle Sink node failure problem, and temporary reliability, Scalable and other aspects of the problem ${ }^{[2]}$.

The network consists of $\mathrm{N}$ randomly deployed sensor nodes, which are distributed in a rectangular space of LXW, where I >> M. The application scenario is cyclical data collection. The network has the following properties: the convergence point (DS) is located outside network, and its computing power and energy are not restricted. Sensor node isomorphism, its data fusion function, can perceive their own residual energy, each node has a full network unique identification (ID); the sensor node and DS in the deployment of the location are not cattle move; The node can control the transmission power of the node, and the node can calculate the transmitter power to the approximate distance according to the intensity of the received signal. The node transmits the l-bit data to the position where the distance, the energy consumed by the transmission circuit loss and power amplifier loss composed of two parts.

Wireless Network monitoring technology achieves low-cost, short-range, low throughput, low power, low speed, high security of transmission, remote monitoring and other fields, which has a wide range of applications, while the terminal nodes can be equipped with various sensors, and remote positioning. Both can be artificially controlled according to the actual situation of the network structure, as well as free and flexible networking, network forms, simple structure, coupled with the low cost, ideal for use in harsh environments, nobody enters the work environment. Meanwhile, there are also testing technology to fill the gaps of low-rate wireless sensor networks, and enrich the content of the radio transmission technology ${ }^{[3]}$. Fig. 1 shows protocol structure of wireless monitoring.

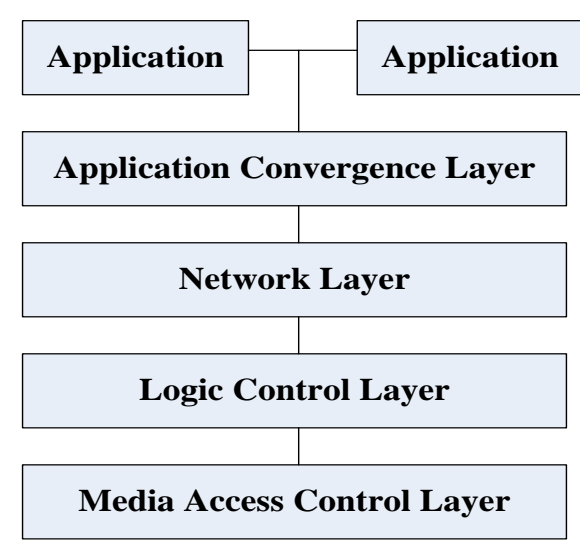

Fig. 1.The protocol structure of wireless monitoring

\section{Energy Consumption Model}

The energy consumption of the node consists of three parts: the energy consumption of the transmitted data Et, the energy consumption of the received data Er, and energy consumption of the data fusion Ec. Assume that each node in the network to measure the surrounding environment after the need to transmit the data length is $\mathrm{k}$ bit, data fusion is that each node can receive it to the $\mathrm{n}$ sub-nodes sent over $\mathrm{n} \mathrm{k}$ bit data and its own $\mathrm{k} \mathrm{Bi} \mathrm{t} \mathrm{data,} \mathrm{the} \mathrm{formation} \mathrm{of} \mathrm{k}$ bi t data sent to its parent node ${ }^{[5]}$.

An energy consumption graph is a weighted graph that characterizes the energy required to send data to each node of the sensor network. The definition point set $\mathrm{v}$ contains the nodes and base stations B of the network. When the distance between nodes: which is less than the communication 
radius of the node, the weight of the $\mathrm{V}$ and $\mathrm{V}$ weights in the energy consumption graph $\mathrm{G}$ is equal to the node's data from $v$ to vi, the required energy Consumption. The energy tree is a weighting tree that characterizes the communication energy and communication routes of each node of the sensor network. V represents the network node set, E represents the edge set, W represents the weight of the edge, $\mathrm{C}$ represent the communication energy consumption of the node, contains the transmission energy ET, and receives the energy consumption ER, the data fusion energy consumption EC. Assuming node $\mathrm{v}$, there are $\mathrm{m}$, sub-nodes, then communication power consumption. Fig. 2 shows energy consumption charts and energy consumption trees.
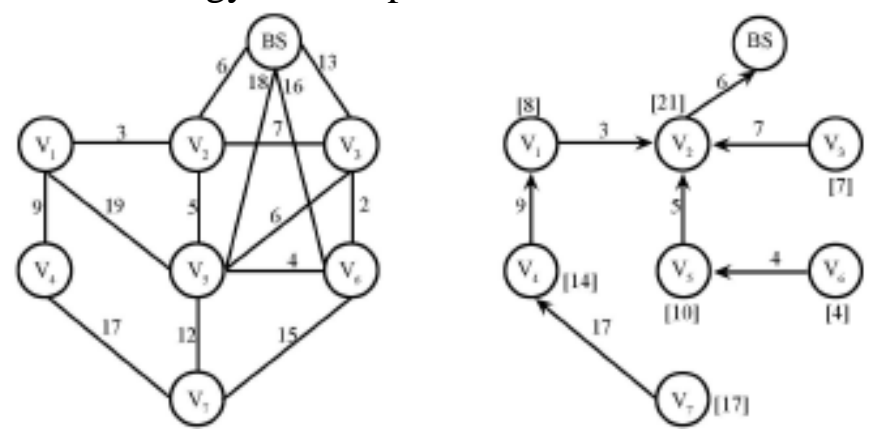

Fig. 2.The energy consumption charts and energy consumption trees

Distributed W RT algorithm, n nodes in the network to find the nearest neighbor node, by judging the comparison of each node and its corresponding neighbor node energy consumption, as far as possible to reduce the maximum energy consumption of the node power to select the appropriate node, through the choice of $n$ rounds of the construction of weighting tree, the formation of network nodes and base stations of the communication route. Each node of the energy tree sends data to its parent node. After receiving the data of the child node, the parent node passes the data with its own data and gets the k bit data and then passes it to its parent node. Finally, all the data are arrival of the base station BS node next to the square brackets marked a round of communication process.

\section{Equilibrium Model of Energy Consumption}

Energy tree algorithm performs the entire judgment tree process by the base station BS, reducing the energy cost of the node in constructing the communication route. By constructing a centralized energy tree with the base station BS as the root node and each node as the tree node, the data transmission between the network nodes is realized, and the node lifetime is extended as much as possible. The algorithm assumes that each node is stationary and the base station BS knows the location of each node. The initial energy of each node is the same, the base station BS energy infinite. After each round of communication, the base station BS knows the remaining energy of each node at present. Fig.3 shows equilibrium model of energy consumption.
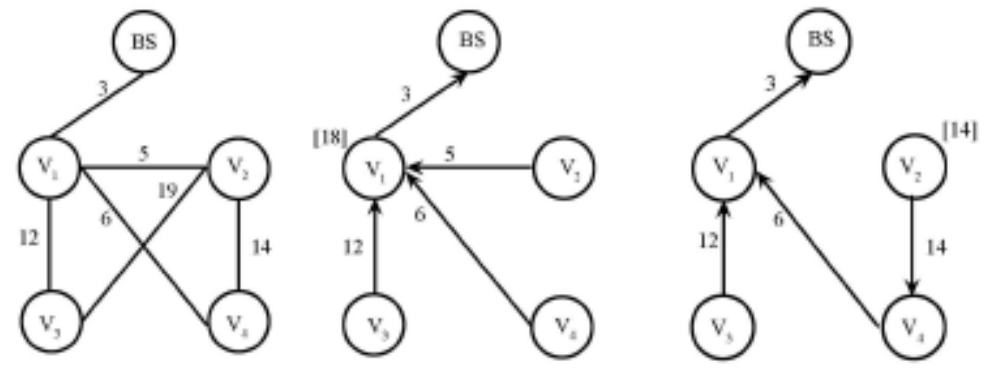

Fig. 3.The equilibrium model of energy consumption

Firstly, the CCT algorithm is used by the base station BS to complete the construction of the virtual energy tree. The virtual energy tree only records the communication route between the network nodes. The nodes do not establish the actual communication link, and then try to adjust the local communication Power consumption of the largest point of power, get better results. After comparing the selection of the optimal result, the base station BS establishes the communication 
route according to the optimal result. The whole tree process uses the known location of the network node to construct different virtual energy consumption tree and make local adjustment by the base station BS. After the optimal result, each network node establishes the connection and communicates according to the route. For each node in the network, the optimal route is chosen without increasing its power consumption.

The centralized energy tree algorithm is: step 1: The base station BS uses the centralized energy consumption tree algorithm to virtualize the virtual energy consumption tree to find the maximum energy consumption point. Step 2: rebuild the CCT algorithm to construct the virtual energy tree, and the difference between the first construction and the node. When looking for a neighbor, look for the smaller value in the ownership value and start the node. Step 3: re-use the CCT algorithm to construct the virtual energy tree, and the difference from the first construction. When looking for a neighbor to the node, find the sub-value in the weight value so that the node starts. Also record the maximum energy consumption. Step 4: compare the size of the node, select the smallest of the corresponding structure of the corresponding structure to improve energy tree. Using the improved centralized energy tree algorithm, we can get the energy tree with closer to the global optimal solution and get the maximum power consumption of the smaller energy consumption.

\section{Conclusions}

In this paper, a new clustering algorithm for energy load balancing is proposed. The new algorithm divides the energy according to the node into several groups, and dynamically adjusts the number of packets to control the size of the clusters, and reduces the energy load when the node energy is low. Secondly, the energy center of gravity as a basis for selection, distributed throughout the network more uniform, while the introduction of energy fast rotation mechanism and energy multi-hop routing to balance the energy load. The new algorithm can significantly prolong stable network, shortening the period of instability, load balancing energy node, the network has fast convergence, so the new algorithm is more in line with characteristic WSN improve the overall performance of the network. Reducing the energy cost and time overhead of the packet is the next step to be studied.

\section{References}

[1] Jennifer Yick, Biswanath M ukherjee, Dipak Ghosal. Wireless Sensor Network Survey [J].Computer Networks 52(2008): 2292- 2320.

[2] FAPOJUWO A, Carro T. Energy consumption and message delay analysis of Cao enhanced base station controlled dynamic clustering protocol for wireless sensor networks [J]. IEEE Transactions on Wireless Communications, 2009, 8(10): 5366-5374.

[3] Lm Y, Xiong N, ZHAO Y. Multi-layer clustering routing algorithm for wireless vehicular sensor networks [J]. IET Communications, 2010, 4(7): 810-816.

[4] Rahman K C. A Survey on Sensor Network [J]. Journal of Computer and information, 2010, 1 (1):76-87.

[5] LI XLNBO, Ll NA, et al. An improved LLACII for clustering protocols in wireless sensor networks [C] // ICMTMA 2010: International Conference on Measuring Technology and Mechatronics Automaton. Washington, DC: IEEE Computer Society, 2010: 496-499. 